\title{
Commodity Futures Hedging, Risk Aversion and the Hedging Horizon
}

\author{
Thomas Conlon ${ }^{\mathrm{a}}$, John Cotter $^{\mathrm{a}}$, Ramazan Gençay ${ }^{\mathrm{b}}$ \\ ${ }^{a}$ Smurfit School of Business, University College Dublin, Carysfort Avenue, Blackrock, \\ Co. Dublin, Ireland. \\ ${ }^{b}$ Department of Economics, Simon Fraser University, 8888 University Drive, Burnaby, \\ British Columbia, V5A 1S6, Canada.
}

\begin{abstract}
This paper examines the impact of investor preferences on the optimal futures hedging strategy and associated hedging performance. Explicit risk aversion levels are often overlooked in hedging analysis. Applying a mean-variance hedging objective, the optimal futures hedging ratio is determined for a range of investor preferences on risk aversion, hedging horizon and expected returns. Wavelet analysis is applied to illustrate how investor time horizon shapes hedging strategy. Empirical results reveal substantial variation of the optimal hedge ratio for distinct investor preferences and are supportive of the hedging policies of real firms. Hedging performance is then shown to be strongly dependent on underlying preferences. In particular, investors with high levels of risk aversion and a short horizon reduce the risk of the hedge portfolio but achieve inferior utility in comparison to those with low risk aversion. Keywords: Commodity Markets, Futures Hedging, Risk Aversion, Hedging Horizon, Wavelet Analysis, Selective Hedging.

EFMA Classification Codes: 420 ; 450
\end{abstract}

Email Addresses: conlon.thomas@ucd.ie (Thomas Conlon - corresponding author), john.cotter@ucd.ie (John Cotter), gencay@sfu.ca (Ramazan Gençay).

The authors would like to acknowledge the financial support of Science Foundation Ireland under Grant Number 08/SRC/FM1389.

January 10, 2013 


\section{Introduction}

Large commodity price fluctuations have increased the importance of hedging to both producers and consumers. ${ }^{1}$ Derivative securities, in particular futures contracts, allow the hedging of operational risks associated with commodity usage. Risk minimization objectives are often considered in the literature, to determine the optimal number of futures contracts necessary to remove the risk associated with holding a spot position in a given commodity. ${ }^{2}$ However, research suggests that the assumptions underlying minimum variance hedging may be relaxed in practise, with hedging strategies shaped by both the level of managerial risk aversion and their view on future market returns (Géczy et al., 2007; Tufano, 1996). Moreover, the horizon of the investor has been shown to alter the optimal hedging strategy (In \& Kim, 2006). By incorporating a range of specific investor preferences using a mean-variance hedging framework, this paper illustrates how investor preferences impact the optimal futures hedging strategy and associated performance. The horizon dependent mean-variance hedging approach is shown to result in hedge ratios that vary substantially in magnitude, in keeping with findings for the hedging policies of real firms (Haushalter, 2000; Tufano, 1996).

Two alternative theories exist, as to the motivation behind corporate risk management activities (Jin \& Jorion, 2006). The first suggests that managers attempt to maximize shareholder value by hedging, but mixed empirical support for the maximization theory exists. ${ }^{3}$ The second theory suggests that management undertake hedging strategies in order to diversify their personal portfolio or to maximize their personal utility, perhaps driven by firm compensation structure. Stulz (1984) demonstrated the theoretical importance of the

\footnotetext{
${ }^{1}$ The higher cost of commodity inputs, such as cotton, and the impact on firm profitability was discussed in the Economist (2011). Producers are having difficulty in passing rises in commodity prices onto consumers, increasing the importance of hedging in the management of firms operational and cash-flow risks.

${ }^{2}$ Recent contributions to the futures hedging literature include a regime switching GARCH model with conditional jump dynamics, Lee (2009), the effect of asymmetric basis on bivariate GARCH models of commodity hedging, Lien \& Yang (2008) and the impact of basis convergence and long memory on the optimal minimum-variance futures hedge, Dark (2007).

${ }^{3}$ Jin \& Jorion (2006) find that hedging reduces exposure to underlying prices but does not affect market value of the firm; Allayannis (2001) finds evidence of a positive relation between firm value and the use of foreign currency derivatives, while Tufano (1996) finds little evidence for the value maximization hypothesis.
} 
level of managerial risk aversion to the optimal hedging strategy, with greater managerial risk tolerance leading to speculative positions. Several empirical studies have also addressed the link between operational risk management, managerial incentives and risk aversion. ${ }^{4}$ Given the evidence for the importance of managerial risk aversion in corporate risk management, in this paper we explicitly incorporate risk aversion preferences in the calculation of the optimal futures hedging strategy. In the empirical literature varying levels of investor risk aversion have been demonstrated, motivating the study of different risk aversion preferences in the case of futures hedging studied here (Bekaert et al., 2009).

Recent studies of corporate risk management have documented evidence that hedging programmes of firms are influenced by the market views of management. 5 Depending on management's expectations about future market prices, the size and timing of hedges may vary. Stulz (1996) suggested that firms may have private information relating to their market and that this may influence the extent to which they hedge. The evidence for selective hedging is in contrast to the hedging literature focused on a risk minimisation objective, with empirical results showing hedging strategy influenced by management's view on expected returns (Brown et al., 2006). In contrast, the minimum-variance objective assumes that returns follow a pure martingale process with zero expected returns. Given that management may believe that they have access to privileged information allowing them to predict future returns, it is important to understand how their optimal hedging strategy varies from the risk minimisation objective with differing private information. In this paper, a range of nonzero expected return assumptions are incorporated using a mean-variance hedging objective, resulting in substantial variation of the optimal hedge ratio and supportive of the hedging policies of real firms.

\footnotetext{
${ }^{4}$ Tufano (1996) and Haushalter (2000) demonstrate that certain firms choose to hedge none of their production, while others choose to hedge a proportion of commodity risk driven in part by managerial risk aversion.

${ }^{5}$ In the case of gold mining firms, Brown et al. (2006) demonstrated that gold producers hedge selectively, increasing their hedge ratios as gold prices decrease. Géczy et al. (2007) studied the tendency of firms to "take a view" on interest rate and currency movements, suggesting that perceived information and cost advantages lead them to hedge actively. Considerable evidence of selective hedging was found by Adam \& Fernando (2006), with only small marginal cash flow gains to firms.
} 
Further, important features of financial data such as volatility and correlation, have been extensively documented to have specific characteristics for the time horizon examined. Considering the interaction between risk aversion and investor horizon, Barberis (2000) demonstrated increased optimal stock allocation in a portfolio context at long horizons. For the case of futures hedging the optimal minimum-variance hedge ratio has been shown to increase for data sampled at longer horizons, with a corresponding increase in hedge performance (Ederington, 1979). However, sampling data at increasing horizons suffers from sample reduction problems associated with reduced quantities of data. To overcome this problem, recent studies have introduced wavelet multiscaling techniques and we follow this approach here. ${ }^{6}$ Wavelet decomposition facilitates an examination of different investor horizons, by breaking a time-series into constituent time horizons and incorporating information at each horizon that would be excluded using a sub-sampling method.

In the futures hedging literature, In \& Kim (2006) describe how wavelets allow the determination of the unique minimum-variance hedge ratio associated with different hedging horizons for the S\&P 500. The optimal hedge ratio and associated hedging effectiveness were shown to increase at long time horizons, with both converging to one (implying a fully hedged position gives full risk protection at long horizons). ${ }^{7}$ In the present work, we build on previous work by calculating a horizon dependent mean-variance hedge ratio using the wavelet transform. This explicitly incorporates preferences on both risk aversion and selective hedging in the calculation of the optimal horizon dependent futures hedge ratio and the associated hedging performance.

Considering the described empirical evidence for the influence of risk aversion, selective hedging and investor horizon in firm hedging strategy, it is important to examine the im-

\footnotetext{
${ }^{6}$ Wavelet multiscaling techniques have been applied to numerous problems in Finance, such as the analysis of high frequency foreign exchange data (Ramsey \& Zhang, 1997) and changes in systematic risk over different time horizons (Gençay et al., 2005). Wavelets have also been used to derive insight into relationships between financial assets at different time-horizons (In et al., 2011; Rua \& Nunes, 2009; Kim \& In, 2005). Further details on the application of wavelets to financial research can be found in Gençay et al. (2001).

${ }^{7}$ Further, Fernandez (2008) and Lien \& Shrestha (2007) applied wavelet decomposition to minimumvariance futures hedging, demonstrating improved hedging performance for investors with long time horizons.
} 
plications for the futures hedging strategy adopted by firms and the performance achieved. This paper makes a number of important contributions to the literature: First, we show how substantial cross-sectional variation in hedge ratios can emerge, driven by differing risk aversion and time horizon preferences of firm management in a mean-variance framework. ${ }^{8}$ The hedge ratios are contrasted with traditional risk minimizing minimum-variance ratios, with investor preferences shown to alter the optimal hedge ratio considerably.

Assumptions on selective hedging due to private information are then incorporated in a mean-variance framework, by testing expected return assumptions with varying magnitude and direction. The interaction between different hedging preferences are examined, with speculative hedging strategies resulting for hedgers with low risk aversion, long time horizons and non-zero expected returns. The insights obtained complement those found in previous studies focused on a simple horizon dependent minimum-variance hedging strategy (Lien \& Shrestha, 2007; In \& Kim, 2006). Finally, given the evidence of diverse hedging strategies among real firms we assess the hedging performance achieved by hedgers with differing preferences. Contrasting hedging performance is demonstrated from risk minimization and utility perspectives, with performance shown to be strongly dependent on the underlying preferences of the hedger. In particular, hedgers with high levels of risk aversion and a short horizon are shown to reduce the risk of the hedge portfolio but achieve inferior utility in comparison to those with low risk aversion. Thus, the implication for firm hedging strategy is that both optimal hedge ratios and associated performance are dependent upon the preferences of the hedgers.

The remainder of this paper is organized as follows: Section 2 presents the methodology used to derive the optimal hedge strategy incorporating differing risk aversion, time-horizon and private information preferences. Section 3 discusses the commodity data examined and presents the empirical results. Concluding comments are given in Section 4 .

\footnotetext{
${ }^{8}$ The importance of risk aversion and time horizon in a mean-variance setting, although not in the context of futures hedging, have also been studied in optimal trade execution (Huberman \& Stanzl, 2005; Almgren, 2003; Holden \& Subrahmanyam, 1996).
} 


\section{Methodology}

\subsection{Optimal Futures Hedge Ratio}

The optimal futures hedge ratio depends on the objective function to be optimized, with many different objective functions proposed, such as minimum-variance, mean-Gini and generalized semivariance. ${ }^{9}$ In this paper, we follow an expected utility framework, in order to capture the impact of differing hedger preferences on the optimal hedge ratio. Assuming the hedger has a long spot position in the underlying commodity, the return, $r_{h}$, on the hedge portfolio is given by

$$
r_{h}=r_{s}-h r_{f}
$$

where $r_{s}$ and $r_{f}$ are the log returns of the spot and futures prices respectively, and $h$ is the hedge ratio. Throughout the hedging literature, a mean-variance expected utility function is often adopted, (Alizadeh et al., 2008; Brooks et al., 2002; Gagnon et al., 1998; Kroner \& Sultan, 1993), an approach we follow here. ${ }^{10}$ If $E\left(r_{h}\right)$ and $\operatorname{var}\left(r_{h}\right)$ are the expected return and variance of the hedge portfolio respectively, then the expected utility function is given by

$$
E U\left(r_{h}\right)=E\left(r_{h}\right)-\operatorname{\alpha var}\left(r_{h}\right)
$$

where $\alpha>0$ is a risk aversion parameter. The hedger then maximizes expected utility,

$$
\begin{aligned}
\max _{h} E U\left(r_{h}\right)= & \max _{h}\left[E\left(r_{s}\right)-h E\left(r_{f}\right)\right. \\
& \left.-\alpha\left(\operatorname{var}\left(r_{s}\right)+h^{2} \operatorname{var}\left(r_{f}\right)-2 h \operatorname{cov}\left(r_{s}, r_{f}\right)\right)\right],
\end{aligned}
$$

leading to an optimal hedge ratio, $h$, of futures contracts to hedge the spot position,

$$
h=\frac{\operatorname{cov}\left(r_{s}, r_{f}\right)}{\operatorname{var}\left(r_{f}\right)}-\frac{E\left(r_{f}\right)}{2 \alpha \operatorname{var}\left(r_{f}\right)},
$$

where $\operatorname{cov}\left(r_{s}, r_{f}\right)$ is the covariance between spot and futures returns, $\operatorname{var}\left(r_{f}\right)$ the variance of futures returns and $E\left(r_{f}\right)$ the expected futures return.

\footnotetext{
${ }^{9}$ See Chen et al. (2003) for a throughout review of different objective functions applied in the futures hedging literature.

${ }^{10}$ The mean-variance approximation to expected utility is only precisely consistent with the expected utility hypothesis in the case of quadratic utility and normal asset returns. However, Kroll et al. (1984); Levy \& Markowitz (1979) showed similar portfolio decisions for a wide range of alternative utility functions.
} 
If the hedger has infinite risk aversion, $\alpha=\infty$, or futures prices follow a martingale, then (5) reduces to the first term, corresponding to a variance minimizing hedge ratio (Ederington, 1979). ${ }^{11}$ The second term in (5) is the speculative component, whose relative importance increases as risk aversion decreases. Given the recent evidence that firms adopt selective hedging, management may assume non-zero expected return driven by their proprietary information. ${ }^{12}$ Depending upon their risk aversion preferences and expected returns assumptions, the hedging strategy adopted by management may differ from the minimum-variance strategy. In this paper the effect of varying expected returns assumptions on the optimal hedge ratio are assessed for a set of both positive and negative expected returns for different management risk aversion levels and hedging horizons. This contributes additional insights beyond the horizon dependent minimum-variance hedging strategy previously detailed, (Lien \& Shrestha, 2007; In \& Kim, 2006), by capturing additional hedging preferences.

\subsection{Hedging Performance}

The primary motivation to test the performance of a hedging strategy is to ensure that risk is reduced in the expected fashion. Dependent on the objective of the hedger, a variety of methods to measure the performance of a hedging strategy have been proposed. In the case of a minimum-variance hedge, Ederington (1979), the hedging performance can be measured as the fraction of portfolio variance removed by hedging, ${ }^{13}$

$$
H E_{\text {variance }}=1-\frac{\operatorname{Variance}\left(\tilde{r}_{h}\right)}{\operatorname{Variance}\left(\tilde{r}_{s}\right)},
$$

where $\tilde{r}_{h}$ and $\tilde{r}_{s}$ are the out-of-sample returns for the hedge and spot portfolios respectively.

While the variance reduction performance measure captures the risk of the portfolio, it fails to consider the trade-off between risk and return, important to a hedger with a view on expected returns driven by private information. In order to consider the risk-return trade-off, we also measure the out-of-sample utility of the portfolio, assuming the same mean-variance

\footnotetext{
${ }^{11}$ The minimum-variance hedge ratio was compared to a full expected utility maximization, (Lence, 1996), with optimal minimum-variance hedge ratios shown to be substantially different from mean-variance hedges.

${ }^{12}$ In the context of portfolio optimisation, Best \& Grauer (1991) examined the sensitivity of portfolio weights to changes in the expected returns of assets.

${ }^{13}$ This is also given by the $R^{2}$ from a regression analysis, Ederington (1979).
} 
utility function described above. Mean-variance utility has been commonly adopted as a measure of hedging performance (Lee, 2009; Lien \& Yang, 2008; Gagnon et al., 1998; Kroner \& Sultan, 1993). By measuring performance using the same risk aversion level as assumed in the calculation of the mean-variance hedge ratio, we give a consistent view of the hedger's preferences. The out-of-sample utility is given by,

$$
U\left(\tilde{r}_{h}\right)=\tilde{r}_{h}-\alpha \operatorname{var}\left(\tilde{r}_{h}\right)
$$

where the risk aversion level, $\alpha$, corresponds to the level used in the calculation of the optimal hedge ratio, (5), and $\tilde{r}_{h}$ is the realized out-of-sample return of the hedge portfolio.

We have now defined the tools necessary to model changes in the optimal hedge ratio and associated performance for hedgers with differing preferences. Next, we detail the use of wavelet multiscale analysis in the determination of changes in the optimal hedge ratio at different time horizons.

\subsection{Wavelet Multiscale Analysis}

Financial and economic time series may have differing empirical characteristics as a function of time horizon. Wavelets provide an efficient means of studying the multi-horizon properties of time-series as they can be used to decompose a signal into different time horizons or frequency components. Further, wavelets help to overcome the sample reduction problem normally found for low-frequency data, capturing information associated with all available data. An alternative to the wavelet analysis is the Fourier transform, which uses a set of sine and cosine functions to transform a time-series from the time to the frequency domain, but does not preserve the information in time. The wavelet transform is applied here due to its better localization properties and ability to handle non-stationary data, important in the context of financial data.

A wavelet is a small wave which grows and decays in a limited time period. ${ }^{14}$ To formalize the notion of a wavelet, let $\psi($.$) be a real valued function with integral zero,$

$$
\int_{-\infty}^{\infty} \psi(t) d t=0
$$

\footnotetext{
${ }^{14}$ The contrasting notion is a big wave such as the sine function which keeps oscillating indefinitely.
} 
Further, the square of the function integrates to unity,

$$
\int_{-\infty}^{\infty} \psi(t)^{2} d t=1
$$

Wavelets are, in particular, useful for the study of how weighted averages vary from one averaging period to the next. Let $x(t)$ be a real-valued function and consider the integral

$$
\bar{x}(s, e) \equiv \frac{1}{e-s} \int_{s}^{e} x(u) d u
$$

where we assume that $e>s . \bar{x}(s, e)$ is the average value of $x($.$) over the interval [s, e]$. Instead of treating an average value $\bar{x}(s, e)$ as a function of end points of the interval $[s, e]$, it can be considered as a function of the length of the interval,

$$
\lambda \equiv e-s
$$

while centering the interval at

$$
t=(s+e) / 2
$$

$\lambda$ is referred to as the time horizon ${ }^{15}$ associated with the average, and using $\lambda$ and $t$ the average can be redefined such that

$$
a(\lambda, t) \equiv \bar{x}\left(t-\frac{\lambda}{2}, t+\frac{\lambda}{2}\right)=\frac{1}{\lambda} \int_{t-\frac{\lambda}{2}}^{t+\frac{\lambda}{2}} x(u) d u
$$

where $a(\lambda, t)$ is the average of $x($.$) over a time horizon of \lambda$ centered at time $t$. The change in $a(\lambda, t)$ from one period to another is measured by

$$
w(\lambda, t) \equiv a\left(\lambda, t+\frac{\lambda}{2}\right)-a\left(\lambda, t-\frac{\lambda}{2}\right)=\frac{1}{\lambda} \int_{t}^{t+\lambda} x(u) d u-\frac{1}{\lambda} \int_{t-\lambda}^{t} x(u) d u .
$$

This measures how much the average changes between two adjacent nonoverlapping time intervals, from $t-\lambda$ to $t+\lambda$, each with a length of $\lambda$. As the two integrals in equation (14) involve nonoverlapping intervals, they can be combined into a single integral over the real axis to obtain,

$$
w(\lambda, t)=\int_{-\infty}^{\infty} \tilde{\psi}(t) x(u) d u
$$

\footnotetext{
${ }^{15}$ Also referred to as the scale associated with the average.
} 
where

$$
\tilde{\psi}(t)= \begin{cases}\frac{-1}{\lambda}, & t-\lambda<u<t, \\ \frac{1}{\lambda}, & t<u<t+\lambda, \\ 0, & \text { otherwise. }\end{cases}
$$

$w(\lambda, t)$ are the wavelet coefficients and they are essentially the changes in averages across adjacent (weighted) averages. Further mathematical details on the wavelet transform can be found in Appendix A.

\subsection{Wavelet variance and covariance}

An important application of the wavelet transform is the ability to decompose the variance of a time-series at different horizons. This is possible as the total variance can be shown to be invariant between the transformed and original process (see Appendix A for further details). For each of the moments necessary in the calculation of the optimal mean-variance hedge ratio, (5), an analogous horizon dependent moment can be calculated using wavelets. The wavelet coefficients, $w_{f}(\lambda, t)$ and $w_{g}(\lambda, t)$, associated with a particular time horizon $\lambda$ and time $t$ for functions $f$ and $g$ can be used to calculate a horizon dependent wavelet covariance (Percival \& Walden, 2000).

An unbiased ${ }^{16}$ estimator of the wavelet covariance at time horizon $\lambda_{j}=2^{j-1}$ is given by

$$
\operatorname{cov}_{f g}\left(\lambda_{j}\right)=\frac{1}{M_{j}} \sum_{t=L_{j}-1}^{N-1} w_{f}\left(\lambda_{j}, t\right) w_{g}\left(\lambda_{j}, t\right)
$$

where $M_{j}=N-L_{j}+1$ is the number of coefficients remaining after discarding the boundary coefficients. The wavelet variance for function $f$ at a particular time horizon $\lambda_{j}$ is similarly defined,

$$
\operatorname{var}_{f}\left(\lambda_{j}\right)=\frac{1}{M_{j}} \sum_{t=L_{j}-1}^{N-1}\left[w_{f}\left(\lambda_{j}, t\right)\right]^{2} .
$$

The wavelet variance and covariance decompose the statistics of a financial time-series at increasingly higher resolutions and allow the exploration of the signal at different time hori-

\footnotetext{
${ }^{16}$ At a time-series boundary, the wavelet transform uses 'mirrored' coefficients, potentially introducing a bias to the data. To alleviate this, the coefficients affected by the boundary are removed from the calculation of the statistics. Biased coefficients were also tested with little quantitative implication for our results.
} 
zons. ${ }^{17}$ In the case of the commodity hedgers studied, the original data is of monthly horizon, leading to wavelet variance-covariance at $1-2$ month, $2-4$ month, $4-8$ month, $8-16$ month and $16-32$ month horizons, incorporating both short- and long-run horizons. ${ }^{18}$

The horizon dependent wavelet covariance, (17), and variance, (18), can then be applied to calculate a time horizon dependent version of the optimal mean-variance hedge ratio (5),

$$
h\left(\lambda_{j}\right)=\frac{\operatorname{cov}\left(r_{f}, r_{s} ; \lambda_{j}\right)}{\operatorname{var}\left(r_{f} ; \lambda_{j}\right)}-\frac{E\left(r_{f}\right)}{2 \alpha \operatorname{var}\left(r_{f} ; \lambda_{j}\right)} .
$$

where $\operatorname{cov}\left(r_{f}, r_{s} ; \lambda_{j}\right)$ corresponds to the covariance between the spot and futures returns, and $\operatorname{var}\left(r_{f} ; \lambda_{j}\right)$ the variance of the futures returns at horizon $\lambda_{j}$. This mean-variance hedge ratio is now dependent on the time horizon, risk aversion and expected return assumptions of the hedger, allowing us to examine how changes in specific hedger preferences alter the optimal hedging strategy.

\section{Data and Empirical Results}

\subsection{Data and Descriptive Statistics}

Monthly spot and futures prices for a range of commodities traded on different exchanges and with diverse fundamental drivers were selected for the study. Coffee (traded on NYBOT), cotton (NYMEX), corn (CBOT) and crude oil (NYMEX) prices from January 1986 through December 2010, a total of 300 months, were obtained from Datastream. In the context of futures hedging, monthly data has been explored previously in a number of studies due to a reduction in nonsynchroneity problems between futures and cash (Adam-Müller \& Nolte, 2011; Ederington \& Salas, 2008). Monthly data is appropriate in this study, as it allows contrast to studies of corporate finance hedging policy where the reported data ranges from monthly horizon up to five years (Jin \& Jorion, 2006; Allayannis \& Ofek, 2001; Haushalter,

\footnotetext{
${ }^{17}$ Confidence intervals for the wavelet variance and covariance may be calculated using large sample theory. See Percival \& Walden (2000) for a detailed treatise.

${ }^{18}$ Throughout this paper, the average time horizon is referred to instead of the range described. Using the average horizon, the monthly commodity returns are decomposed into returns with horizon 1.5, 3, 6, 12 and 24 months.
} 
2000). Each futures contract is nearest-to-maturity and rolled to the new contract on the first day of the contract month.

To decompose the data for both the cash and futures returns into the constituent timehorizons of the investor, the maximum overlap discrete wavelet transform was employed (MODWT, Section A.2). For this study, we selected the least asymmetric (LA) wavelet, (also known as the Symlet, Percival \& Walden (2000)), with filter width LA8, where 8 refers to the length of the scaling function. ${ }^{19}$ The Symlet was chosen as it exhibits near symmetry about the midpoint and allows accurate alignment of wavelet coefficients with the original time-series. In the calculation of the hedge ratio and the associated performance measures, only coefficients unaffected by the boundary are used, eliminating boundary problems.

In order to test the performance of the prescribed hedging strategy at each risk aversion level and time horizon, the time-series is split into two equal segments, where the first is used to calculate the optimal hedge ratio in-sample and the second to test the performance of the strategy out-of-sample (Lee, 2009). At each time horizon, the out-of-sample performance captures the average performance realized by an investor with that horizon, rather than the real-time hedging performance. In-sample performance was also considered, but little additional insight was revealed as full information regarding returns are available to the investor in-sample. ${ }^{20}$ Out-of-sample effectiveness results in a clearer, unbiased view of the performance for hedgers with differing views on expected returns.

The mean-variance hedging objective, (5), requires the hedger view on expected futures returns. In this paper, we examine changes in the hedge ratio for different investor expected return assumptions. To this end, zero expected returns (a pure martingale process) are tested resulting in a minimum-variance hedge. Given the observed tendency for firms to hedge selectively, a range of non-zero expected returns are also examined to determine the impact on commodity futures hedging. Expected returns were chosen with reference to the

\footnotetext{
${ }^{19}$ Different filter widths and wavelet types were also examined. The filter width was chosen to strike a balance between the level of periodicity captured and the highest level of decomposition available. Various other wavelet types, such as the Coiflet and Daubauchies were tested giving little qualitative difference in results. Results, not shown for conciseness, are available from the authors.

${ }^{20}$ In-sample results are not shown for brevity; However, they are available from the authors.
} 
average annualized returns achieved in both the in-sample and out-of-sample periods. Insample, the average returns tended to be negative, while out-of-sample annual returns were found to be positive. The expected returns selected here vary from $-4 \%$ to $+4 \%$, within the range of returns found over the periods studied.

\subsection{Hedging and Risk Aversion}

We first investigate the univariate impact of differing risk aversion preferences on the optimal hedging strategy, using the original unfiltered monthly commodity returns. The optimal mean-variance hedge ratio, (5), is calculated for different values of risk aversion corresponding to low risk aversion $(\alpha=1)$, moderate risk aversion $(\alpha=3)$, high risk aversion $(\alpha=6,10)$ and extremely high risk aversion $(\alpha=1000) .{ }^{21}$ The extremely high risk aversion case approximates the case of infinite risk aversion, equivalent to the minimum-variance portfolio. $^{22}$

The optimal mean-variance hedge ratio for a hedger with varying risk aversion and expected returns preferences is shown in Table 1. For each commodity a range of different expected return assumptions from $-4 \%$ to $+4 \%$ are examined. ${ }^{23}$ For extremely high levels of risk aversion, $(\alpha=1000)$, the optimal hedge ratio is the same across all expected return values since the magnitude of the speculative component in (5) converges to zero. However, for low levels of risk aversion, the optimal hedge ratios diverge significantly, increasing

\footnotetext{
${ }^{21}$ Various levels of risk aversion have been examined in the hedging literature, with Lence (1996) testing values of 1, 3 and 10, Gagnon et al. (1998) examining values between 0.5 and 4, and Kroner \& Sultan (1993) assuming a value of 4 . The range of assumptions for the risk aversion are also in line with the empirical literature, with Bekaert et al. (2009) finding a median risk aversion level of 2.52 but a mean of 7.06.

${ }^{22}$ In the case of extremely high risk aversion, the second term of equation (5) approaches zero, approximating the minimum-variance case. Since the minimum-variance case assumes zero expected return, the extremely high risk aversion case provides an indifferent hedge ratio to a zero expected return mean-variance hedge. Note, we discuss this case implicitly by examining the extremely high risk aversion case, $\alpha=1000$.

${ }^{23}$ Each of these values corresponds to an annualized expected return, chosen to reflect the view of a hedger with additional information they believe not to be available to the market. An example might be the information available to a large oil producer due to knowledge of a refinery due to be taken off-line due to repairs. Additional expected return values were also considered, resulting in some quantitative changes to the hedge ratio but little change to the qualitative implications.
} 
monotonically as the expected return moves from positive to negative. For risk aversion level $\alpha=1$, a hedger expecting a negative return will tend to over-hedge, with the hedge ratio much greater than one. In contrast, the optimal hedge ratio for a positive expected return is lower than the naive (or one-to-one) hedge ratio, ranging from 0.09 to 0.81 across the different commodities studied.

[Table 1 about here.]

For greater risk aversion levels, the optimal hedge ratio decreases (increases) in the case of negative (positive) expected returns, converging to the minimum-variance hedge in each case. At the highest risk aversion levels, the speculative component of (5) is minimized with less importance placed on the private information available to the hedger and more on risk reduction. Previous application of the mean-variance hedging framework, (Rolfo, 1980), assumed a single expected return and varying risk aversion levels. By examining a range of expected return assumptions, we demonstrate the importance of the market view realized from the manager's private information. Hedge ratios greater than one are found only for negative expected returns, corresponding to a risk tolerant hedger taking a speculative position to benefit from their view on future expected return. The actual hedge ratios found previously for the oil and gold-mining industries were rarely greater than one, (Jin \& Jorion, 2006; Haushalter, 2000; Tufano, 1996), suggesting that management rarely speculated on negative expected returns. Empirically, Haushalter (2000) found that oil and gas producers with an active hedging policy hedged an average of $30 \%$ of one year production. Comparing this to the optimal crude oil hedge ratio in table 1, would suggest a very low level of management risk aversion level $(<1)$ and positive expected return assumptions. However, the above analysis assumes that all hedgers have similar hedging horizons, while different producers and consumers may actually have very diverse horizons. We consider the implications of this in the following sections.

\subsection{Futures Hedging and the Hedging Horizon}

In order to calculate the horizon dependent variance of and covariance between futures and spot, each commodity time-series is first decomposed into constituent time horizons 
using the maximum overlap discrete wavelet transform (MODWT - See appendix A.2 for further details). Table 2 presents the variance, covariance and correlation statistics for each commodity at different average time horizons corresponding to 1.5, 3, 6, 12 and 24 months. The variance at each horizon corresponds to the contribution to total sample variance and is found to peak at the shortest time horizon for all assets. With the exception of Corn (which peaks at medium time-horizons), the maximum covariance is also found at short horizon. Also shown in table 2 are correlations between spot and futures at different horizons. In the cases of cotton, corn and crude oil correlation is found to increase at longer time horizons, in keeping with findings for other assets in previous studies (Fernandez, 2008; Lien \& Shrestha, 2007; In \& Kim, 2006). Coffee is the exception here, with maximum correlation found at intermediate horizon, perhaps explained by difficulties in coffee storage over longer horizons.

[Table 2 about here.]

The minimum-variance hedge ratio for each asset is also shown in Table 2, with larger hedge ratios at longer time horizons in keeping with the findings of Fernandez (2008); Lien \& Shrestha (2007); In \& Kim (2006). At the shortest horizon of 1.5 months, the optimal minimum-variance hedge ratio ranges from 0.33 in the case of cotton to 0.99 for crude oil. At the longest time horizon studied, the hedge ratio ranges from 0.82 (coffee) to 1.08 (cotton). Considering the different assets, a wide divergence is found with a range of 0.75 between hedge ratios at the shortest and longest horizons for Cotton but negligible in the case of crude oil, possibly reflecting the liquidity of each market, (crude oil being the most widely traded commodity). The hedging performance at each horizon is also examined for all assets, measured using variance reduction and is found to increase at longer horizons. Having separately considered the impact of risk aversion, hedging horizon and speculative hedging on the optimal hedging strategy, we next incorporate assumptions on all to examine their impact on commodity hedging strategy.

\subsection{Hedging, Risk Aversion and the Hedging Horizon}

Having examined the change in the optimal futures hedge ratio for differing risk aversion, selective hedging and time horizon separately in previous sections, here we consider a horizon 
dependent mean-variance framework that incorporates all. Table 3 considers the optimal mean-variance hedge ratio for hedgers with different hedging horizons, risk aversion attitudes and expected return assumptions. ${ }^{24}$ First, we detail the results for a hedger assuming positive expected return of $+2 \%$ where, moving from high to low risk aversion, we find a decrease in the optimal hedge ratio for all assets. This is in keeping with the premise that an investor with low risk aversion has higher risk tolerance and is concerned with both risk and return. These risk tolerant investors are willing to reduce the size of their hedge to gain a positive expected return. Looking down the different time horizons in table 3 for an investor with extremely low risk aversion $(\alpha=1)$, we find that the optimal hedge ratio decreases at longer horizon taking on negative values at a 24 month horizon, for all assets examined. This suggests that it is optimal for an investor with a long time horizon and low risk aversion to take a speculative position (reverse hedging) using futures. ${ }^{25}$ However, empirical studies have found little evidence for reverse hedging in the case of gold miners or oil and gas firms, (Jin \& Jorion, 2006; Haushalter, 2000; Tufano, 1996), suggesting a zero lower bound on the hedge ratio at each horizon. This, in turn, implies a lower bound on the risk aversion level of the hedger at each horizon, with a lower bound between 2 and 4 imputed across the assets studied, in keeping with that found in the asset pricing literature (Mehra \& Prescott, 1985).

[Table 3 about here.]

Next, we consider the optimal hedge ratio for a negative expected return, $-2 \%$. In contrast to the results for positive expected returns, the optimal hedge ratio is found to increase moving from high to low risk aversion levels. The hedger, in order to profit from the expected direction of the commodity, takes a larger short position in the futures market for low risk aversion levels. In keeping with the findings for positive expected return, at long

\footnotetext{
${ }^{24}$ The average hedge ratio and $95 \%$ confidence interval were also calculated using a bootstrap method and are available from the authors. Results show that the hedge ratios found are within the $95 \%$ confidence intervals from the bootstrap.

${ }^{25}$ This result is consistent with the findings of Barberis (2000), where the optimal equity allocation for long horizon investors were shown to be larger than for short horizon investors due to predictability in equity returns.
} 
horizons the optimal hedge ratio for low risk aversion levels diverges from the one-to-one or naive hedge, resulting in a net short speculative position in futures. ${ }^{26}$

At very high risk aversion levels, $(\alpha=1000)$, the hedge ratio converges to the minimumvariance hedge ratio, regardless of the expected return assumption. In contrast to previous findings, (Fernandez (2008) and references therein), the maximal hedge ratio is found at intermediate horizon for certain assets, (coffee, corn and crude oil). However, the underlying data examined here is of a much longer horizon, (monthly rather than daily in previous studies), contributing additional insight from the perspective of longer-term hedgers.

For robustness, differing expected returns assumptions of $-4 \%$ and $+4 \%$ are also studied for each commodity, with the optimal hedge ratio in each case shown in table 4 . The trends in the hedge ratio for different horizons and risk aversion assumptions are similar to those found in table 3 for smaller magnitude expected returns. Naturally, for very high levels of risk aversion, $\alpha=1000$, the ratio is identical, as the speculative component of (5) is negligible. At low levels of risk aversion, $\alpha=1, \ldots, 3$, the divergence between the hedge ratio for positive and negative returns is more pronounced than that found earlier. At these levels, the risk tolerant hedger is attempting to increase their expected future wealth with less regard for the level of risk involved. Further, at long hedging horizons the speculative component dominates, with the hedger taking larger positions to benefit further from the expected bias in the return and the lower relative volatility, driven by mean-reversion in commodities (Schwartz, 1997). Comparing to the previous results for different returns assumptions, the direction of expected return is important at all horizons, while the magnitude of expected return has relatively more impact on the optimal hedging strategy at longer horizon.

[Table 4 about here.]

Given the evidence that risk aversion, selective hedging and hedging horizon influence the hedging strategy of real firms, we have shown that incorporating each into an optimal hedging model produces a wide range of hedge ratios, in keeping with that found for real firms (Haushalter, 2000; Tufano, 1996). As an example, consider a minimum-variance hedge

\footnotetext{
${ }^{26}$ Note that a positive number in table 3 implies that the hedger sells short that number of contracts, (5), while a negative number corresponds to a long speculative position in the futures.
} 
for a crude oil producer, which would suggest a hedge ratio approaching one. In a study of oil and gas producers from 1992 to 1994, Haushalter (2000) found that for companies that hedge production, on average only $30 \%$ of one year production was hedged. In our analysis, to achieve a hedge ratio like that found empirically at a twelve month horizon the hedger would require a relatively low risk aversion level $(\alpha=1, \ldots, 3)$ and a positive expected return assumption. This suggests that minimum-variance hedging, although prevalent throughout the literature, may not capture the true preferences of hedgers. Incorporating risk aversion, through mean-variance hedging, in combination with the hedging horizon allows for a richer variety of preferences and a better understanding of the impact on hedging strategy. This, in turn, helps us to understand the impact of managerial preferences on the operations of the firm.

\subsection{Hedging Performance}

Incorporating investor preferences on risk aversion, hedging horizon and expected return (selective hedging) into a mean-variance hedging objective, a wide range of possible hedge ratios were detailed across all commodities studied. Given the diverse hedge ratios demonstrated here and the evidence for wide ranging hedge ratios adopted by real firms, it is important to understand the performance achieved by each hedging strategy. Dependent on the objective of the hedger each of the ratios prescribed will also deliver varying hedging performance. In order to determine the performance of the optimal hedging strategy across different risk aversion levels and wavelet derived hedging horizons, we consider two different performance measures. Performance is tested out-of-sample, with half the data used to calculate the relevant hedge ratio and the remaining data used for out-of-sample assessment.

In table 5 performance is measured using the traditional variance reduction method, with performance shown for a range of risk aversion levels and hedging horizons. Similar to the case of an investor who hedges at a weekly or monthly period (Ederington, 1979), our results show the average performance achieved by a hedger at different horizons. Results are shown for two levels of expected return, $\pm 2 \%$, for the hedge ratios corresponding to table $3 .^{27}$ First, we examine the variance risk reduction performance corresponding to the

\footnotetext{
${ }^{27}$ Effectiveness results for expected returns of $\pm 4 \%$ were also studied and were found to be qualitatively
} 
original unfiltered returns with small variation found for differing risk aversion level. Across the different wavelet transformed horizons, for very high levels of risk aversion the hedge ratio for hedgers expecting both positive and negative return is equal, resulting in identical effectiveness. For high levels of risk aversion hedging performance is found to be improved at longer horizons, with large performance increases in particular for coffee and cotton.

At low risk aversion levels, the performance varies markedly across assets. In the case of crude oil, similar performance is found for positive and negative expected returns, while for cotton the performance at short horizons differs greatly. Across all assets, at long horizons we find an increase in the hedge portfolio variance relative to the spot position for low levels of risk aversion, driven by the hedger's willingness to increase the expected return of the portfolio with little focus on the risk of the hedge portfolio. This corresponds to a hedger taking a speculative position, expecting mean reversion in commodities with little focus on risk (Schwartz, 1997). However, little empirical evidence for this 'reverse hedging' exists in the literature, suggesting higher risk aversion preferences in practise (Haushalter, 2000; Tufano, 1996).

[Table 5 about here.]

The application of variance-reduction as a measure of hedging performance captures only the reduction in spot portfolio risk. However, a hedger assuming a non-zero expected futures return may want to consider the trade-off between risk and return in evaluating the performance of the hedging strategy. A common approach to incorporate both risk and return in performance measurement is to evaluate the mean-variance utility of the hedge portfolio. ${ }^{28}$ While previous studies have applied a minimum-variance hedging approach and measured performance using mean-variance utility, (Alizadeh et al., 2008; Fernandez, 2008; In \& Kim, 2006), we provide a consistent view by applying the same hedging preferences to find both the optimal mean variance hedge ratio and measure associated performance.

The out-of-sample utility, (7), of the hedge portfolio can be found in table 6 for hedge

similar. Results are not presented for conciseness but are available on request.

${ }^{28}$ The economic benefits from hedging have been commonly measured using mean-variance utility (Lee, 2009; Lien \& Yang, 2008; In \& Kim, 2006; Gagnon et al., 1998; Kroner \& Sultan, 1993). 
ratios corresponding to those in table 3. In agreement with the findings of In \& Kim (2006); Fernandez (2008) the utility of the hedge portfolio is found to be negative for a wide range of scales and horizons. For all assets at the shortest horizons, a monotonic decrease in the realized utility is found at greater risk aversion levels across both positive and negative expected returns. Compared with low risk aversion hedgers, those having very high risk aversion (minimum-variance hedgers), are giving up expected returns in order to focus on decreasing their risk levels, thus lowering their mean-variance utility. At longer horizons, the out-of-sample utility is found to be greatly dependent on the expected return. In each case, the realized out-of-sample return is positive, resulting in contrasting performance for long horizon positive and negative expected return hedgers. Those with positive (negative) expected returns, experience positive (negative) expected utility at long horizons, decreasing (increasing) at higher risk aversion levels. Moreover, across all risk aversion levels a hedger using unfiltered original data to calculate his hedge ratio achieves a lower level of utility than a hedger focussed on a particular horizon, confirming the importance of considering horizon independent of aversion or expected returns preferences.

[Table 6 about here.]

Comparing the hedging horizons, for very high risk aversion levels (the minimum-variance hedge), the highest utility is achieved for hedgers with a long horizon, with a monotonic increase for all assets. For small values of aversion, the results are again dependent on the expected return. Positive expected return hedgers experience an increase in utility at long horizons, due to a decrease in the size of their hedge position. In contrast, the large speculative hedge positions adopted by negative expected return hedgers result in monotonically decreasing utility at long horizons. These results demonstrate that while low risk aversion hedgers can benefit at longer horizon, their expected return assumptions and associated private information leading to the expected returns are crucial in the outcome. As described previously in the case of a crude oil producer, the hedge ratio may be considerably less than a minimum-variance hedge, resulting in large deviation in performance depending on the accuracy of the expected returns assumptions at low risk aversion levels. At the longest horizons, a hedger correctly anticipating a positive expected return would have benefited 
from having low risk aversion, while a negative expected return hedger would have benefited further at high levels of aversion.

Comparing the different performance metrics studies, high risk aversion hedgers achieve best performance in terms of risk reduction, measured using variance reduction. However, measured using out-of-sample utility, the performance was weakest (strongest) at high risk aversion for negative (positive) expected returns compared to other risk aversion levels. This demonstrates the importance of considering hedge portfolio realized return in measuring the hedging performance, in particular for hedgers with low risk aversion preferences. For high risk aversion hedgers, best performance is found at the longest horizon examined, regardless of the effectiveness measure applied. The implication for firm hedging strategy is that both optimal hedge ratios and associated performance are dependent upon the preference of the hedger.

\section{Conclusions}

In this paper, we examine the impact of differing investor preferences on the optimal futures hedging strategy. Given the evidence that assumptions underlying minimum variance hedging may be relaxed in practise, we investigate how preferences on hedging horizon, risk aversion and selective hedging impact the optimal mean-variance futures hedging strategy and associated hedging performance. The paper provides insight into the interaction between the optimal commodity hedge ratio and investor risk aversion, selective hedging and hedging horizon.

A mean-variance hedging framework is applied in order to examine changes in the optimal futures hedge ratio for a range of preferences. Using wavelets, we transform commodity returns data into different horizons, allowing us to examine a number of different hedging horizons with a limited dataset. The horizon dependent mean-variance hedging approach is shown to result in hedge ratios that vary widely in magnitude for all commodities, in keeping with findings for the hedging policies of real firms (Haushalter, 2000; Tufano, 1996).

For positive expected returns, the optimal hedge ratio is shown to decrease for lower risk aversion levels, as low risk aversion is associated with higher levels of speculation for risk tolerant investors. Comparing long to short horizons, the optimal hedge ratio is significantly 
smaller at low risk aversion levels. In contrast, for negative expected returns the optimal mean-variance hedge ratio is found to increase at low risk aversion levels, with the hedger attempting to benefit from their view of expected returns (due to private information). Again, a sharp contrast is found between short and long horizon hedgers, with long horizon hedgers willing to speculate further. Finally, for extremely high levels of risk aversion, expected returns are of negligible importance to the hedger and so the hedge ratio converges for all returns assumptions. Additional analysis of larger magnitude expected returns demonstrate greater diversity between hedge ratios for differing risk aversion preferences.

Performance of the diverse hedge ratios detailed is then examined, with contrasting performance found from risk minimization and utility perspectives. Measuring the level of variance remaining after hedging, differing results are found, dependent on the interaction between risk aversion and hedging horizon. For high risk aversion, the best risk reduction performance is obtained at long horizons. For low risk aversion levels, the amount of residual risk is found to increase at long horizons, due to the speculative component associated with the hedge ratio. Moreover, the highest levels of utility were found for hedgers who correctly forecasted positive expected returns and had a long horizon. Hedgers with high levels of risk aversion and a short horizon are shown to reduce the risk of the hedge portfolio but achieve inferior utility in comparison to those with low risk aversion. The implication for firm hedging strategy is that both optimal hedge ratios and associated performance are dependent upon the preferences of the investor. 


\section{Appendices}

\section{A. Wavelet Transformations}

\section{A.1. Discrete Wavelet Transform}

In order to capture the optimal hedging characteristics associated with different time horizons, we first decompose each time-series using the wavelet transform. Decomposing the data results in a series of coefficients associated with a particular time horizon and can be performed using the (discrete) wavelet transformation (DWT). Formally, let us introduce the DWT through a simple matrix operation. Let $\mathbf{y}$ be the dyadic length vector $\left(T=2^{J}\right)$ of observations. The length $T$ vector of discrete wavelet coefficients $\mathbf{w}$ is obtained via

$$
\mathbf{w}=\mathcal{W} \mathbf{y}
$$

where $W$ is a $T \times T$ orthonormal matrix defining the DWT. The vector of wavelet coefficients can be organized into $J+1$ vectors, $\mathbf{w}=\left[\mathbf{w}_{1}, \mathbf{w}_{2}, \ldots, \mathbf{w}_{J}, \mathbf{v}_{J}\right]^{\prime}$, where $\mathbf{w}_{j}$ is a length $\frac{T}{2^{j}}$ vector of wavelet coefficients associated with changes on a time horizon of length $\lambda_{j}=2^{j-1}$ and $\mathbf{v}_{J}$ is a length $T / 2^{j}$ vector of scaling coefficients associated with averages on a time horizon of length $2^{J}=2 \lambda_{J}$.

The matrix $\mathrm{W}$ is composed of the wavelet and scaling filter coefficients arranged on a row-by-row basis. Let

$$
\mathbf{h}_{1}=\left[h_{1, N-1}, h_{1, N-2}, \ldots, h_{1,1}, h_{1,0}\right]^{\prime}
$$

be the vector of zero-padded unit horizon wavelet filter coefficients in reverse order. Thus, the coefficients $h_{1,0}, \ldots, h_{1, L-1}$ are taken from an appropriate orthonormal wavelet family of length $L$, and all values $L<t<T$ are defined to be zero. Now circularly shift $\mathbf{h}_{1}$ by factors of two so that

$$
\begin{aligned}
& \mathbf{h}_{1}^{(2)}=\left[h_{1,1}, h_{1,0}, h_{1, N-1}, h_{1, N-2} \ldots, h_{1,3}, h_{1,2}\right]^{\prime} \\
& \mathbf{h}_{1}^{(4)}=\left[h_{1,3}, h_{1,2}, h_{1,1}, h_{1,0} \ldots, h_{1,5}, h_{1,4}\right]^{\prime}
\end{aligned}
$$

and so on. Define the $\frac{T}{2} \times T$ dimensional matrix $\mathcal{W}_{1}$ to be the collection of $\frac{T}{2}$ circularly shifted versions of $\mathbf{h}_{1}$. Hence,

$$
\mathcal{W}_{1}=\left[\mathbf{h}_{1}^{(2)}, \mathbf{h}_{1}^{(4)}, \ldots, \mathbf{h}_{1}^{\left(\frac{T}{2}-1\right)}, \mathbf{h}_{1}\right]^{\prime} .
$$


Let $\mathbf{h}_{2}$ be the vector of zero-padded horizon 2 wavelet filter coefficients defined similarly to $\mathbf{h}_{1}$. $\mathcal{W}_{2}$ is constructed by circularly shifting the vector $\mathbf{h}_{2}$ by factor of four. Repeat this to construct $\mathcal{W}_{j}$ by circularly shifting the vector $\mathbf{h}_{j}$ (the vector of zero-padded horizon $j$ wavelet filter coefficients) by $2^{j}$. The matrix $\mathcal{V}_{J}$ is simply a column vector whose elements are all equal to $\frac{1}{\sqrt{T}}$. Then, the $T \times T$ dimensional matrix $\mathcal{W}$ is $\mathcal{W}=\left[\mathcal{W}_{1}, \mathcal{W}_{2}, \ldots, \mathcal{W}_{J}, \mathcal{V}_{J}\right]^{\prime}$.

When we are provided with a dyadic length time-series, it is not necessary to implement the DWT down to level $J=\log _{2}(T)$. A partial DWT may be performed instead that terminates at level $J_{p}<J$. The resulting vector of wavelet coefficients will now contain $T-\frac{T}{2^{J_{p}}}$ wavelet coefficients and $\frac{T}{2^{J_{p}}}$ scaling coefficients.

The orthonormality of the matrix $W$ implies that the DWT is a variance preserving transformation:

$$
\|\mathbf{w}\|=\sum_{t=1}^{\frac{T}{2^{J}}} v_{t, J}^{2}+\sum_{j=1}^{J}\left(\sum_{t=1}^{\frac{T}{2^{J}}} w_{t, j}^{2}\right)=\sum_{j=1}^{T} y_{t}^{2}=\|y\|^{2}
$$

This can be easily proven through basic matrix manipulation via

$$
\|\mathbf{y}\|^{2}=\mathbf{y}^{\prime} \mathbf{y}=(\mathcal{W} \mathbf{w})^{\prime} \mathcal{W} \mathbf{w}=\mathbf{w}^{\prime} \mathcal{W}^{\prime} \mathcal{W} \mathbf{w}=\mathbf{w}^{\prime} \mathbf{w}=\|\mathbf{w}\|
$$

Given the structure of the wavelet coefficients, $\|y\|^{2}$ is decomposed on a horizon-by-horizon basis by

$$
\|\mathbf{y}\|^{2}=\sum_{j=1}^{J}\left\|w_{j}\right\|^{2}+\left\|v_{j}\right\|^{2}
$$

where $\left\|w_{j}\right\|^{2}=\sum_{t=1}^{\frac{T}{2 j}} w_{t, j}^{2}$ is the sum of squared variation of $y$ due to changes at horizon $\lambda_{j}$ and $\left\|v_{j}\right\|^{2}=\sum_{t=1}^{\frac{T}{2 j}} v_{t, j}^{2}$ is the information due to changes at horizons $\lambda_{J}$ and higher.

\section{A.2. Maximum Overlap Discrete Wavelet Transformation}

An alternative wavelet transform is the maximum overlap discrete wavelet transformation (MODWT) which is computed by not subsampling the filtered output. Let $\mathbf{y}$ be a vector of observations with arbitrary length $\mathrm{T}$. The length $(J+1) T$ vector of MODWT coefficients $\tilde{\mathbf{w}}$ is obtained via

$$
\tilde{\mathbf{w}}=\tilde{\mathcal{W}}_{\mathbf{y}}
$$


where $\tilde{\mathcal{W}}$ is a $(J+1) T \times T$ matrix defining the MODWT. The vector of MODWT coefficients may be organized into $J+1$ vectors via

$$
\tilde{\mathbf{w}}=\left[\tilde{\mathbf{w}}_{1}, \tilde{\mathbf{w}}_{2}, \ldots, \tilde{\mathbf{w}}_{J}, \tilde{\mathbf{v}}_{J}\right]^{T}
$$

where $\tilde{\mathbf{w}}_{j}$ is a length $T$ vector of wavelet coefficients associated with changes on a horizon of length $\lambda_{j}=2^{j-1}$ and $\tilde{\mathbf{v}}_{J}$ is a length $\mathrm{T}$ vector of scaling coefficients associated with averages on a horizon of length $2^{J}=2 \lambda_{J}$, just as with the DWT.

Similar to the orthonormal matrix defining the DWT, the matrix $\tilde{\mathcal{W}}$ is made up of $J+1$ submatrices, each size $T \times T$, and may be expressed as

$$
\tilde{\mathcal{W}}=\left[\begin{array}{l}
\tilde{\mathcal{W}}_{1} \\
\tilde{\mathcal{W}}_{2} \\
\vdots \\
\tilde{\mathcal{W}}_{J} \\
\tilde{\mathcal{V}}_{J}
\end{array}\right]
$$

The MODWT utilizes the rescaled filters $\tilde{\mathbf{h}}_{j}=\frac{\tilde{\mathbf{h}}_{j}}{2^{\frac{j}{2}}}$ and $\tilde{\mathbf{g}}_{J}=\frac{\tilde{\mathbf{h}}_{J}}{2^{\frac{J}{2}}},(j=1, \ldots, J)$. To construct the $T \times T$ dimensional submatrix $\tilde{\mathcal{W}}_{1}$, we circularly shift the rescaled wavelet filter vector $\tilde{\mathbf{h}}_{1}$ by integer units to the right so that

$$
\tilde{\mathcal{W}}_{1}=\left[\tilde{\mathbf{h}}_{1}^{(1)}, \tilde{\mathbf{h}}_{1}^{(2)}, \tilde{\mathbf{h}}_{1}^{(3)}, \ldots, \tilde{\mathbf{h}}_{1}^{(N-2)}, \tilde{\mathbf{h}}_{1}^{(N-1)}, \tilde{\mathbf{h}}_{1}\right]^{T}
$$

This matrix may be interpreted as the interweaving of the DWT submatrix $\mathcal{W}_{1}$ with a circularly shifted (to the right by one unit) version of itself. The remaining submatrices $\tilde{\mathcal{W}}_{2}, \ldots, \tilde{\mathcal{W}}_{J}$ are formed similarly to Equation 29 , only replace $\tilde{\mathbf{h}}_{1}$ with $\tilde{\mathbf{h}}_{j}$.

In practice, a pyramid algorithm is utilized similar to that of the DWT to compute the MODWT. Starting with the data $x_{t}$ (no longer restricted to be a dyadic length), filter it using $\tilde{\mathbf{h}}_{1}$ and $\tilde{\mathbf{g}}_{1}$ to obtain the length $T$ vectors of wavelet and scaling coefficients $\tilde{\mathbf{w}}_{1}$ and $\tilde{\mathbf{v}}_{1}$, respectively.

For each iteration of the MODWT pyramid algorithm, we require three objects: the data vector $\mathbf{x}$, the wavelet filter $\tilde{h}_{l}$ and the scaling filter $\tilde{g}_{l}$. The first iteration of the pyramid algorithm begins by filtering (convolving) the data with each filter to obtain the following 
wavelet and scaling coefficients:

$$
\tilde{w}_{1, t}=\sum_{l=0}^{L-1} \tilde{h}_{l} y_{t-l} \bmod T \quad \text { and } \quad \tilde{v}_{1, t}=\sum_{l=0}^{L-1} \tilde{g}_{l} y_{t-l} \bmod T
$$

where $t=1, \ldots, T$. The length $\mathrm{T}$ vector of observations has been high- and low-pass filtered to obtain T coefficients associated with this information. The second step of the MODWT pyramid algorithm starts by defining the data to be the scaling coefficients $\tilde{\mathbf{v}}_{1}$ from the first iteration and apply the filtering operations as above to obtain the second level of wavelet and scaling coefficients

$$
\tilde{w}_{2, t}=\sum_{l=0}^{L-1} \tilde{h}_{l} \tilde{v}_{1, t-2 l} \bmod T \quad \text { and } \quad \tilde{v}_{2, t}=\sum_{l=0}^{L-1} \tilde{g}_{l} \tilde{v}_{1, t-2 l} \bmod T,
$$

with $t=1, \ldots, T$. Keeping all vectors of wavelet coefficients, and the final level of scaling coefficients, we have the following length $T$ decomposition: $\tilde{\mathbf{w}}=\left[\tilde{\mathbf{w}}_{1}, \tilde{\mathbf{w}}_{2}, \tilde{\mathbf{v}}_{2}\right]^{\prime}$. After the third iteration of the pyramid algorithm, where we apply filtering operations to $\tilde{\mathbf{v}}_{2}$, the decomposition now looks like $\tilde{\mathbf{w}}=\left[\tilde{\mathbf{w}}_{1}, \tilde{\mathbf{w}}_{2}, \tilde{\mathbf{w}}_{3}, \tilde{\mathbf{v}}_{3}\right]^{\prime}$. This procedure may be repeated up to $J$ times where $J=\log _{2}(T)$ and gives the vector of MODWT coefficients in Equation 28.

Similar to DWT, MODWT wavelet and scaling coefficients are variance preserving

$$
\|\tilde{\mathbf{w}}\|^{2}=\sum_{t=1}^{T} \tilde{v}_{t, J}^{2}+\sum_{j=1}^{J}\left(\sum_{t=1}^{T} \tilde{w}_{t, j}^{2}\right)=\sum_{j=1}^{T} y_{t}^{2}=\|\mathbf{y}\|^{2} .
$$

and a partial decomposition $J_{p}<J$ may be performed when it is deemed necessary.

The following properties are important for distinguishing the MODWT from the DWT. The MODWT can accommodate any sample size $T$, while the $J_{p}^{\text {th }}$ order partial DWT restricts the sample size to a multiple of $2^{J_{p}}$. The detail and smooth coefficients of a MODWT are associated with zero phase filters. Thus, events that feature in the original time series can be properly aligned with features in the MODWT multiresolution analysis. The MODWT is invariant to circular shifts in the original time series. This property does not hold for the DWT. The MODWT wavelet variance estimator is asymptotically more efficient than the same estimator based on the DWT. For both MODWT and DWT, the scaling coefficients contain the lowest frequency information. But each level's wavelet coefficients contain progressively lower frequency information. 


\section{References}

Adam, T., \& Fernando, C. (2006). Hedging, speculation, and shareholder value. Journal of Financial Economics, 81, 283-309.

Adam-Müller, A. F., \& Nolte, I. (2011). Cross hedging under multiplicative basis risk. Journal of Banking EG Finance, 35, 2956-2964.

Alizadeh, A., Nomikos, N., \& Pouliasis, P. (2008). A Markov regime switching approach for hedging energy commodities. Journal of Banking \& Finance, 32, 1970-1983.

Allayannis, G. (2001). The use of foreign currency derivatives and firm market value. Review of Financial Studies, 14, 243-276.

Allayannis, G., \& Ofek, E. (2001). Exchange rate exposure, hedging, and the use of foreign currency derivatives. Journal of International Money and Finance, 20, 273-296.

Almgren, R. F. (2003). Optimal execution with nonlinear impact functions and tradingenhanced risk. Applied Mathematical Finance, 10, 1-18.

Barberis, N. (2000). Investing for the long run when returns are predictable. The Journal of Finance, LV, 225-264.

Bekaert, G., Engstrom, E., \& Xing, Y. (2009). Risk, uncertainty, and asset prices. Journal of Financial Economics, 91, 59-82.

Best, M., \& Grauer, R. (1991). On the sensitivity of mean-variance-efficient portfolios to changes in asset means: Some analytical and computational results. Review of Financial Studies, 4, 315-342.

Brooks, C., Henry, O., \& Persand, G. (2002). The effect of asymmetries on optimal hedge ratios. Journal of Business, 75, 333-352.

Brown, G. W., Crabb, P. R., \& Haushalter, D. (2006). Are Firms Successful at Selective Hedging ? Journal of Business, 79, 2925-2949. 
Chen, S., Lee, C., \& Shrestha, K. (2003). Futures hedge ratios: a review. The Quarterly Review of Economics and Finance, 43, 433-465.

Dark, J. (2007). Basis Convergence and Long Memory in Volatility when dynamic hedging with futures. Journal of financial and quantitative analysis, 42, 1021-1040.

Economist, T. (2011). Everyday higher prices. 24th February 2011: http://www.economist.com/node/18231464.

Ederington, L., \& Salas, J. (2008). Minimum variance hedging when spot price changes are partially predictable. Journal of Banking $\&$ Finance, 32, 654-663.

Ederington, L. H. (1979). The hedging performance of the new futures markets. The Journal of Finance, 34, 157.

Fernandez, V. (2008). Multi-period hedge ratios for a multi-asset portfolio when accounting for returns co-movement. Journal of Futures Markets, 28, 182-207.

Gagnon, L., Lypny, G., \& McCurdy, T. (1998). Hedging foreign currency portfolios. Journal of Empirical Finance, 5, 197-220.

Géczy, C., Minton, B., \& Schrand, C. (2007). Taking a View: Corporate Speculation, Governance, and Compensation. The Journal of Finance, 62, 2405-2443.

Gençay, R., Selcuk, B., \& Whitcher, B. (2001). An Introduction to wavelets and other filtering methods in finance and economics. San Diego, CA: Academic Press.

Gençay, R., Selcuk, F., \& Whitcher, B. (2005). Multiscale systematic risk. Journal of International Money and Finance, 24, 55-70.

Haushalter, G. D. (2000). Financing policy, basis risk, and corporate hedging: evidence from oil and gas producers. The Journal of Finance, 55, 107-152.

Holden, C., \& Subrahmanyam, A. (1996). Risk aversion, liquidity, and endogenous short horizons. Review of Financial Studies, 9, 691-722. 
Huberman, G., \& Stanzl, W. (2005). Optimal Liquidity Trading. Review of Finance, 9, $165-200$.

In, F., \& Kim, S. (2006). The hedge ratio and the empirical relationship between the stock and futures markets: A new approach using wavelet analysis. The Journal of Business, $79,799-820$.

In, F., Kim, S., \& Gençay, R. (2011). Investment horizon effect on asset allocation between value and growth strategies. Economic Modelling, 28, 1489-1497.

Jin, Y., \& Jorion, P. (2006). Firm value and hedging: Evidence from U.S. oil and gas producers. The Journal of Finance, 61, 893-919.

Kim, S., \& In, F. (2005). The relationship between stock returns and inflation: new evidence from wavelet analysis. Journal of Empirical Finance, 12, 435-444.

Kroll, Y., Levy, H., \& Markowitz, H. M. (1984). Mean-variance versus direct utility maximization. The Journal of Finance, 39, 47.

Kroner, K., \& Sultan, J. (1993). Time-varying distributions and dynamic hedging with foreign currency futures. Journal of Financial and Quantitative Analysis, 28, 535-551.

Lee, H.-T. (2009). Optimal futures hedging under jump switching dynamics. Journal of Empirical Finance, 16, 446-456.

Lence, S. H. (1996). Relaxing the assumptions of minimum-variance Hedging. Journal of Agricultural and Resource Economics, 21, 39-55.

Levy, H., \& Markowitz, H. (1979). Approximating expected utility by a function of mean and variance. The American Economic Review, 69, 308-317.

Lien, D., \& Shrestha, K. (2007). An empirical analysis of the relationship between hedge ratio and hedging horizon using wavelet analysis. Journal of Futures Markets, 27, 127-150.

Lien, D., \& Yang, L. (2008). Asymmetric effect of basis on dynamic futures hedging: Empirical evidence from commodity markets. Journal of Banking \&3 Finance, 32, 187-198. 
Mehra, R., \& Prescott, E. (1985). The equity premium: A puzzle. Journal of monetary Economics, 15, 145-161.

Percival, D., \& Walden, A. (2000). Wavelet methods for time series analysis. Cambridge: Cambridge University Press.

Ramsey, J., \& Zhang, Z. (1997). The analysis of foreign exchange data using waveform dictionaries. Journal of Empirical Finance, 4, 341-372.

Rolfo, J. (1980). Optimal hedging under price and quantity uncertainty: The case of a cocoa producer. The Journal of Political Economy, 88, 100-116.

Rua, A., \& Nunes, L. (2009). International comovement of stock market returns: A wavelet analysis. Journal of Empirical Finance, 16, 632-639.

Schwartz, E. (1997). The stochastic behavior of commodity prices: Implications for valuation and hedging. Journal of Finance, 52, 923-974.

Stulz, R. (1984). Optimal hedging policies. Journal of Financial and Quantitative Analysis, 19, 127-140.

Stulz, R. (1996). Rethinking risk management. Journal of Applied Corporate Finance, 9 , $8-25$.

Tufano, P. (1996). Who manages risk? An empirical examination of risk management practices in the gold mining industry. Journal of Finance, 51, 1097-1137. 


\begin{tabular}{cccccc}
\hline & \multicolumn{5}{c}{ Risk Aversion } \\
Coffee & 1 & 3 & 6 & 10 & 1000 \\
\cline { 2 - 6 } $\mathrm{E}[\mathrm{r}]=+4 \%$ & 0.62 & 0.77 & 0.81 & 0.82 & 0.85 \\
$\mathrm{E}[\mathrm{r}]=+2 \%$ & 0.73 & 0.81 & 0.83 & 0.84 & 0.85 \\
$\mathrm{E}[\mathrm{r}]=-2 \%$ & 0.96 & 0.89 & 0.87 & 0.86 & 0.85 \\
$\mathrm{E}[\mathrm{r}]=-4 \%$ & 1.08 & 0.92 & 0.89 & 0.87 & 0.85 \\
& & & & & \\
Cotton & 1 & 3 & 6 & 10 & 1000 \\
\cline { 2 - 6 } $\mathrm{E}[\mathrm{r}]=+4 \%$ & 0.09 & 0.34 & 0.41 & 0.43 & 0.47 \\
$\mathrm{E}[\mathrm{r}]=+2 \%$ & 0.28 & 0.41 & 0.44 & 0.45 & 0.47 \\
$\mathrm{E}[\mathrm{r}]=-2 \%$ & 0.66 & 0.53 & 0.50 & 0.49 & 0.47 \\
$\mathrm{E}[\mathrm{r}]=-4 \%$ & 0.84 & 0.59 & 0.53 & 0.51 & 0.47 \\
& & & & & \\
$\mathrm{Corn}$ & 1 & 3 & 6 & 10 & 1000 \\
$\mathrm{E}[\mathrm{r}]=+4 \%$ & 0.32 & 0.74 & 0.85 & 0.89 & 0.95 \\
$\mathrm{E}[\mathrm{r}]=+2 \%$ & 0.64 & 0.85 & 0.90 & 0.92 & 0.95 \\
$\mathrm{E}[\mathrm{r}]=-2 \%$ & 1.27 & 1.06 & 1.01 & 0.98 & 0.95 \\
$\mathrm{E}[\mathrm{r}]=-4 \%$ & 1.59 & 1.16 & 1.06 & 1.02 & 0.95 \\
& & & & & \\
$\mathrm{Crude}$ Oil & 1 & 3 & 6 & 10 & 1000 \\
\cline { 2 - 6 }$[\mathrm{r}]=+4 \%$ & 0.64 & 0.87 & 0.93 & 0.95 & 0.98 \\
$\mathrm{E}[\mathrm{r}]=+2 \%$ & 0.81 & 0.93 & 0.96 & 0.97 & 0.98 \\
$\mathrm{E}[\mathrm{r}]=-2 \%$ & 1.16 & 1.04 & 1.01 & 1.00 & 0.99 \\
$\mathrm{E}[\mathrm{r}]=-4 \%$ & 1.33 & 1.10 & 1.04 & 1.02 & 0.99 \\
\hline
\end{tabular}

Table 1: Optimal mean-variance hedge ratios incorporating different investor preferences for risk aversion and expected return using original unfiltered data.

Notes: Hedge ratios calculated in-sample using data from January 1986 - June 1998. For each asset, various expected annualised return values (E[r]) ranging from $-4 \%$ to $+4 \%$ are examined, selected with reference to average annualised returns. The mean-variance hedge ratio is calculated for a set of risk aversion levels, covering the range of those detailed in the literature. Hedge ratios are calculated using original unfiltered commodity returns, excluding information on the planning horizon of the hedger. 


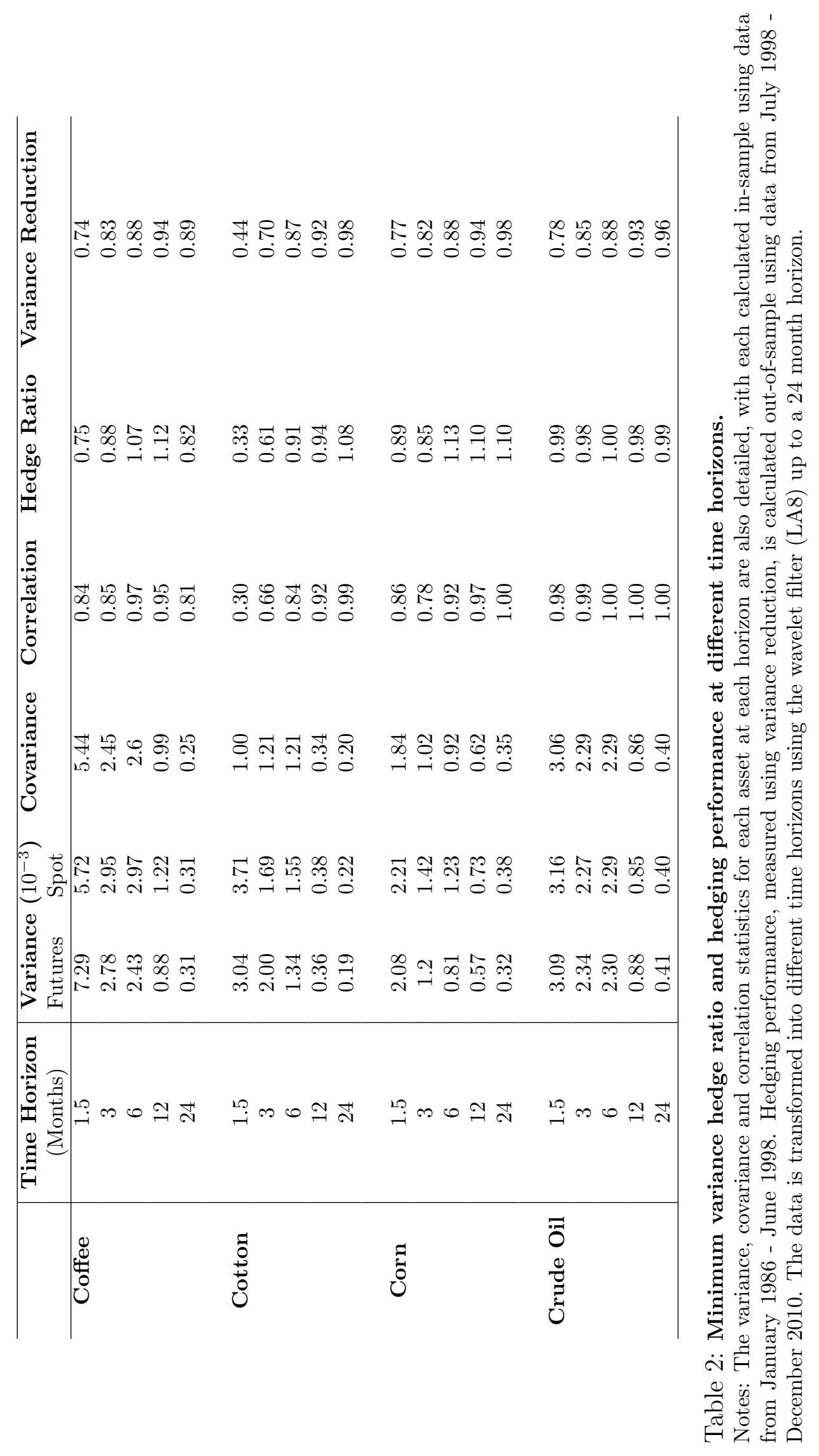




\begin{tabular}{c|ccccc|c} 
Time & \multicolumn{7}{|c}{ Risk Aversion } \\
Horizon & 1 & 3 & 6 & 10 & 1000 & $1000-1$ \\
\hline 1.5 & 0.63 & 0.71 & 0.73 & 0.73 & 0.75 & 0.11 \\
& 0.86 & 0.78 & 0.77 & 0.76 & 0.75 & -0.11 \\
3 & 0.58 & 0.78 & 0.83 & 0.85 & 0.88 & 0.30 \\
& 1.18 & 0.98 & 0.93 & 0.91 & 0.88 & -0.30 \\
6 & 0.73 & 0.96 & 1.01 & 1.04 & 1.07 & 0.34 \\
& 1.41 & 1.18 & 1.13 & 1.10 & 1.07 & -0.34 \\
12 & 0.18 & 0.81 & 0.97 & 1.03 & 1.12 & 0.94 \\
& 2.07 & 1.44 & 1.28 & 1.22 & 1.13 & -0.94 \\
24 & -1.85 & -0.07 & 0.37 & 0.55 & 0.82 & 2.67 \\
& 3.49 & 1.71 & 1.26 & 1.09 & 0.82 & -2.67
\end{tabular}

(a) Coffee

\begin{tabular}{c|ccccc|c} 
Time & \multicolumn{7}{|c}{ Risk Aversion } \\
Horizon & 1 & 3 & 6 & 10 & 1000 & $1000-1$ \\
\hline 1.5 & 0.49 & 0.75 & 0.82 & 0.85 & 0.89 & 0.40 \\
& 1.28 & 1.02 & 0.95 & 0.93 & 0.89 & -0.40 \\
3 & 0.17 & 0.62 & 0.74 & 0.78 & 0.85 & 0.68 \\
& 1.54 & 1.08 & 0.97 & 0.92 & 0.85 & -0.68 \\
6 & 0.12 & 0.79 & 0.96 & 1.03 & 1.13 & 1.02 \\
& 2.15 & 1.47 & 1.30 & 1.23 & 1.13 & -1.02 \\
12 & -0.36 & 0.62 & 0.86 & 0.96 & 1.10 & 1.46 \\
& 2.56 & 1.59 & 1.35 & 1.25 & 1.10 & -1.46 \\
24 & -1.50 & 0.23 & 0.67 & 0.84 & 1.09 & 2.59 \\
& 3.69 & 1.96 & 1.53 & 1.36 & 1.10 & -2.59
\end{tabular}

(c) Corn

\begin{tabular}{c|ccccc|c} 
Time & \multicolumn{7}{|c}{ Risk Aversion } \\
Horizon & 1 & 3 & 6 & 10 & 1000 & $1000-1$ \\
\hline 1.5 & 0.06 & 0.24 & 0.28 & 0.30 & 0.33 & 0.27 \\
& 0.60 & 0.42 & 0.37 & 0.35 & 0.33 & -0.27 \\
3 & 0.19 & 0.47 & 0.54 & 0.57 & 0.61 & 0.41 \\
& 1.02 & 0.75 & 0.68 & 0.65 & 0.61 & -0.41 \\
6 & 0.29 & 0.70 & 0.81 & 0.85 & 0.91 & 0.62 \\
& 1.53 & 1.11 & 1.01 & 0.97 & 0.91 & -0.62 \\
12 & -1.33 & 0.18 & 0.56 & 0.71 & 0.94 & 2.26 \\
& 3.21 & 1.69 & 1.32 & 1.17 & 0.94 & -2.26 \\
24 & -3.38 & -0.41 & 0.33 & 0.63 & 1.07 & 4.45 \\
& 5.53 & 2.56 & 1.82 & 1.52 & 1.08 & -4.45
\end{tabular}

(b) Cotton

\begin{tabular}{c|ccccc|c} 
Time & \multicolumn{7}{|c}{ Risk Aversion } \\
Horizon & 1 & 3 & 6 & 10 & 1000 & $1000-1$ \\
\hline 1.5 & 0.72 & 0.90 & 0.95 & 0.96 & 0.99 & 0.27 \\
& 1.26 & 1.08 & 1.03 & 1.02 & 0.99 & -0.27 \\
3 & 0.63 & 0.86 & 0.92 & 0.94 & 0.98 & 0.35 \\
& 1.33 & 1.10 & 1.04 & 1.01 & 0.98 & -0.35 \\
6 & 0.64 & 0.88 & 0.94 & 0.96 & 1.00 & 0.36 \\
& 1.36 & 1.12 & 1.06 & 1.03 & 1.00 & -0.36 \\
12 & 0.04 & 0.67 & 0.83 & 0.89 & 0.98 & 0.94 \\
& 1.92 & 1.30 & 1.14 & 1.08 & 0.98 & -0.94 \\
24 & -1.03 & 0.32 & 0.65 & 0.79 & 0.99 & 2.02 \\
& 3.01 & 1.66 & 1.33 & 1.19 & 0.99 & -2.02
\end{tabular}

(d) Crude Oil

Table 3: Optimal mean-variance hedge ratios for varying time horizon (months) and risk aversion, with annualised expected returns of $\pm 2 \%$.

Notes: Hedge ratios calculated in-sample using data from January 1986 - June 1998. At each time horizon, the first line corresponds to an annualised expected return $(\mathrm{E}[\mathrm{r}])$ value of $+2 \%$ while the second line corresponds to an annualised expected return of $-2 \%$, selected with reference to average annualised returns. The mean-variance hedge ratio is calculated for a set of risk aversion levels, covering the range of those detailed in the literature. The data is transformed into different time horizons using the wavelet filter (LA8) up to a 24 month horizon. 


\begin{tabular}{c|ccccc|c} 
Time & \multicolumn{7}{|c}{ Risk Aversion } \\
Horizon & 1 & 3 & 6 & 10 & 1000 & $1000-1$ \\
\hline 1.5 & 0.52 & 0.67 & 0.71 & 0.72 & 0.75 & 0.23 \\
& 0.97 & 0.82 & 0.78 & 0.77 & 0.75 & -0.22 \\
3 & 0.29 & 0.68 & 0.78 & 0.82 & 0.88 & 0.59 \\
& 1.47 & 1.08 & 0.98 & 0.94 & 0.88 & -0.59 \\
6 & 0.4 & 0.85 & 0.96 & 1.00 & 1.07 & 0.67 \\
& 1.74 & 1.3 & 1.18 & 1.14 & 1.07 & -0.67 \\
12 & -0.75 & 0.5 & 0.81 & 0.94 & 1.12 & 1.87 \\
& 3.00 & 1.75 & 1.44 & 1.31 & 1.13 & -1.87 \\
24 & -4.49 & -0.95 & -0.07 & 0.29 & 0.81 & 5.30 \\
& 6.12 & 2.59 & 1.7 & 1.35 & 0.82 & -5.30
\end{tabular}

(a) Coffee

\begin{tabular}{c|ccccc|c} 
Time & \multicolumn{7}{|c}{ Risk Aversion } \\
Horizon & 1 & 3 & 6 & 10 & 1000 & $1000-1$ \\
\hline 1.5 & 0.1 & 0.62 & 0.75 & 0.81 & 0.89 & 0.79 \\
& 1.68 & 1.15 & 1.02 & 0.97 & 0.89 & -0.79 \\
3 & -0.51 & 0.4 & 0.62 & 0.72 & 0.85 & 1.36 \\
& 2.21 & 1.31 & 1.08 & 0.99 & 0.85 & -1.36 \\
6 & -0.89 & 0.46 & 0.8 & 0.93 & 1.13 & 2.02 \\
& 3.15 & 1.81 & 1.47 & 1.33 & 1.13 & -2.02 \\
12 & -1.8 & 0.14 & 0.62 & 0.81 & 1.10 & 2.9 \\
& 4.00 & 2.07 & 1.59 & 1.39 & 1.11 & -2.89 \\
24 & -4.06 & -0.62 & 0.24 & 0.58 & 1.09 & 5.15 \\
& 6.25 & 2.82 & 1.96 & 1.61 & 1.10 & -5.15
\end{tabular}

(c) Corn

\begin{tabular}{c|ccccc|c} 
Time & \multicolumn{7}{|c}{ Risk Aversion } \\
Horizon & 1 & 3 & 6 & 10 & 1000 & $1000-1$ \\
\hline 1.5 & -0.21 & 0.15 & 0.24 & 0.27 & 0.33 & 0.54 \\
& 0.87 & 0.51 & 0.42 & 0.38 & 0.33 & -0.54 \\
3 & -0.21 & 0.33 & 0.47 & 0.53 & 0.61 & 0.82 \\
& 1.43 & 0.88 & 0.74 & 0.69 & 0.61 & -0.82 \\
6 & -0.32 & 0.5 & 0.7 & 0.79 & 0.91 & 1.23 \\
& 2.14 & 1.32 & 1.11 & 1.03 & 0.91 & -1.23 \\
12 & -3.57 & -0.56 & 0.19 & 0.49 & 0.93 & 4.50 \\
& 5.44 & 2.44 & 1.69 & 1.39 & 0.94 & -4.50 \\
24 & -7.78 & -1.88 & -0.40 & 0.19 & 1.07 & 8.85 \\
& 9.94 & 4.03 & 2.55 & 1.96 & 1.09 & -8.85
\end{tabular}

(b) Cotton

\begin{tabular}{c|ccccc|c} 
Time & \multicolumn{7}{|c}{ Risk Aversion } \\
Horizon & 1 & 3 & 6 & 10 & 1000 & $1000-1$ \\
\hline 1.5 & 0.46 & 0.81 & 0.90 & 0.94 & 0.99 & 0.53 \\
& 1.52 & 1.17 & 1.08 & 1.04 & 0.99 & -0.53 \\
3 & 0.28 & 0.74 & 0.86 & 0.91 & 0.98 & 0.70 \\
& 1.68 & 1.21 & 1.09 & 1.05 & 0.98 & -0.70 \\
6 & 0.28 & 0.76 & 0.88 & 0.93 & 1.00 & 0.72 \\
& 1.71 & 1.23 & 1.12 & 1.07 & 1.00 & -0.71 \\
12 & -0.89 & 0.36 & 0.67 & 0.8 & 0.98 & 1.87 \\
& 2.85 & 1.6 & 1.29 & 1.17 & 0.98 & -1.87 \\
24 & -3.02 & -0.35 & 0.32 & 0.59 & 0.99 & 4.01 \\
& 5.00 & 2.33 & 1.66 & 1.39 & 0.99 & -4.01
\end{tabular}

(d) Crude Oil

Table 4: Optimal mean-variance hedge ratios for varying time horizon (months) and risk aversion, with annualised expected returns of $\pm 4 \%$.

Notes: Hedge ratios calculated in-sample using data from January 1986 - June 1998. At each time horizon, the first line corresponds to an annualised expected return (E[r]) value of $+4 \%$ while the second line corresponds to an annualised expected return of $-4 \%$, selected with reference to average annualised returns. The mean-variance hedge ratio is calculated for a set of risk aversion levels, covering the range of those detailed in the literature. The data is transformed into different time horizons using the wavelet filter (LA8) up to a 24 month horizon. 


\begin{tabular}{c|ccccc} 
Time & \multicolumn{5}{c}{ Risk Aversion } \\
Horizon & 1 & 3 & 6 & 10 & 1000 \\
\hline Original & 0.73 & 0.75 & 0.75 & 0.75 & 0.75 \\
& 0.75 & 0.76 & 0.76 & 0.76 & 0.75 \\
1.5 & 0.69 & 0.73 & 0.73 & 0.74 & 0.74 \\
& 0.77 & 0.75 & 0.75 & 0.75 & 0.74 \\
3 & 0.68 & 0.8 & 0.82 & 0.82 & 0.83 \\
& 0.84 & 0.85 & 0.84 & 0.84 & 0.83 \\
6 & 0.81 & 0.88 & 0.89 & 0.89 & 0.88 \\
& 0.77 & 0.87 & 0.88 & 0.88 & 0.88 \\
12 & 0.29 & 0.88 & 0.93 & 0.93 & 0.94 \\
& 0.18 & 0.84 & 0.91 & 0.92 & 0.94 \\
24 & -5.5 & -0.12 & 0.52 & 0.7 & 0.89 \\
& -2.77 & 0.79 & 0.98 & 0.98 & 0.89
\end{tabular}

\begin{tabular}{c|ccccc} 
Time & \multicolumn{5}{c}{ Risk Aversion } \\
Horizon & 1 & 3 & 6 & 10 & 1000 \\
\hline Original & 0.44 & 0.60 & 0.63 & 0.64 & 0.66 \\
& 0.81 & 0.72 & 0.69 & 0.68 & 0.66 \\
1.5 & 0.10 & 0.40 & 0.46 & 0.49 & 0.52 \\
& 0.80 & 0.63 & 0.58 & 0.55 & 0.52 \\
3 & 0.34 & 0.7 & 0.76 & 0.79 & 0.82 \\
& 0.97 & 0.91 & 0.87 & 0.85 & 0.82 \\
6 & 0.48 & 0.89 & 0.94 & 0.96 & 0.98 \\
& 0.74 & 0.98 & 0.99 & 0.99 & 0.98 \\
12 & -4.14 & 0.32 & 0.78 & 0.90 & 0.99 \\
& -3.25 & 0.61 & 0.93 & 0.98 & 0.99 \\
24 & -16.66 & -0.92 & 0.53 & 0.84 & 1.00 \\
& -17.04 & -1.05 & 0.47 & 0.80 & 1.00
\end{tabular}

(a) Coffee

\begin{tabular}{c|ccccc} 
Time & \multicolumn{5}{c}{ Risk Aversion } \\
Horizon & 1 & 3 & 6 & 10 & 1000 \\
\hline Original & 0.76 & 0.87 & 0.89 & 0.89 & 0.90 \\
& 0.88 & 0.91 & 0.91 & 0.90 & 0.90 \\
1.5 & 0.65 & 0.84 & 0.88 & 0.89 & 0.90 \\
& 0.90 & 0.93 & 0.92 & 0.91 & 0.90 \\
3 & 0.27 & 0.79 & 0.86 & 0.89 & 0.92 \\
& 0.80 & 0.96 & 0.95 & 0.94 & 0.92 \\
6 & 0.20 & 0.93 & 0.98 & 0.99 & 0.98 \\
& -0.10 & 0.83 & 0.93 & 0.96 & 0.98 \\
12 & -0.78 & 0.82 & 0.96 & 0.98 & 0.99 \\
& -0.98 & 0.75 & 0.92 & 0.96 & 0.99 \\
24 & -4.70 & 0.38 & 0.85 & 0.94 & 0.99 \\
& -4.95 & 0.3 & 0.8 & 0.92 & 0.99
\end{tabular}

(c) Corn

\begin{tabular}{c|ccccc}
\multicolumn{1}{c}{$\begin{array}{c}\text { Time } \\
\text { Horizon }\end{array}$} & 1 & 3 & 6 & 10 & 1000 \\
\hline Original & 0.94 & 0.97 & 0.97 & 0.97 & 0.97 \\
& 0.93 & 0.96 & 0.97 & 0.97 & 0.97 \\
1.5 & 0.92 & 0.98 & 0.99 & 0.99 & 0.99 \\
& 0.91 & 0.98 & 0.98 & 0.99 & 0.99 \\
3 & 0.87 & 0.98 & 0.99 & 1.00 & 1.00 \\
& 0.87 & 0.98 & 1.00 & 1.00 & 1.00 \\
6 & 0.87 & 0.99 & 1.00 & 1.00 & 1.00 \\
& 0.86 & 0.98 & 0.99 & 1.00 & 1.00 \\
12 & 0.08 & 0.89 & 0.97 & 0.99 & 1.00 \\
& 0.13 & 0.91 & 0.98 & 0.99 & 1.00 \\
24 & -3.12 & 0.53 & 0.88 & 0.96 & 1.00 \\
& -3.06 & 0.56 & 0.89 & 0.96 & 1.00
\end{tabular}

(d) Crude Oil

Table 5: Out-of-sample hedging performance, measured using variance reduction, for varying time horizon (months) and risk aversion.

Notes: Out-of-Sample performance over the period July 1998 to December 2010 is measured using the in-sample hedge ratios detailed in table 3. At each time horizon, the first line is the performance assuming an expected return of $+2 \%$ in calculating the hedge ratio, while the second line refers to an expected return of $-2 \%$. The data is transformed into different time horizons using the wavelet filter (LA8) up to a 24 month horizon. 


\begin{tabular}{c|ccccc}
$\begin{array}{c}\text { Time } \\
\text { Horizon }\end{array}$ & 1 & 3 & 6 & 10 & 1000 \\
\hline Original & -0.004 & -0.011 & -0.021 & -0.035 & -3.451 \\
& -0.005 & -0.011 & -0.021 & -0.035 & -3.451 \\
1.5 & -0.001 & -0.003 & -0.007 & -0.011 & -1.057 \\
& -0.001 & -0.003 & -0.006 & -0.011 & -1.057 \\
3 & 0.000 & -0.001 & -0.003 & -0.004 & -0.344 \\
& -0.002 & -0.002 & -0.003 & -0.004 & -0.343 \\
6 & 0.000 & -0.001 & -0.002 & -0.002 & -0.097 \\
& -0.003 & -0.002 & -0.002 & -0.002 & -0.097 \\
12 & 0.002 & -0.000 & -0.001 & -0.001 & -0.038 \\
24 & -0.006 & -0.003 & -0.002 & -0.002 & -0.038 \\
& 0.009 & 0.002 & 0.001 & 0.000 & -0.044 \\
& -0.013 & -0.004 & -0.002 & -0.002 & -0.042
\end{tabular}

(a) Coffee

\begin{tabular}{c|ccccc} 
Time & \multicolumn{5}{|c}{ Risk Aversion } \\
Horizon & 1 & 3 & 6 & 10 & 1000 \\
\hline Original & 0.001 & -0.003 & -0.008 & -0.015 & -1.577 \\
& -0.004 & -0.005 & -0.009 & -0.015 & -1.577 \\
1.5 & 0.003 & 0.000 & -0.002 & -0.004 & -0.497 \\
& -0.002 & -0.001 & -0.002 & -0.003 & -0.495 \\
3 & 0.005 & 0.002 & 0.000 & -0.001 & -0.241 \\
& -0.004 & -0.001 & -0.000 & -0.001 & -0.240 \\
6 & 0.007 & 0.002 & 0.001 & 0.000 & -0.025 \\
& -0.010 & -0.004 & -0.003 & -0.002 & -0.025 \\
12 & 0.011 & 0.003 & 0.002 & 0.001 & -0.007 \\
& -0.014 & -0.005 & -0.003 & -0.002 & -0.007 \\
24 & 0.021 & 0.007 & 0.003 & 0.002 & -0.002 \\
& -0.024 & -0.008 & -0.004 & -0.003 & -0.002
\end{tabular}

(c) Corn

\begin{tabular}{c|ccccc} 
Time & \multicolumn{5}{|c}{ Risk Aversion } \\
Horizon & 1 & 3 & 6 & 10 & 1000 \\
\hline Original & -0.005 & -0.022 & -0.047 & -0.081 & -8.396 \\
1.5 & -0.007 & -0.023 & -0.048 & -0.081 & -8.396 \\
& 0.002 & -0.004 & -0.011 & -0.020 & -2.237 \\
3 & 0.002 & -0.002 & -0.008 & -0.017 & -2.234 \\
& 0.003 & 0.001 & -0.000 & -0.002 & -0.380 \\
6 & -0.000 & 0.001 & 0.000 & -0.001 & -0.378 \\
& 0.004 & 0.001 & 0.001 & 0.000 & -0.040 \\
12 & -0.004 & -0.001 & -0.000 & -0.000 & -0.039 \\
24 & 0.011 & 0.003 & 0.002 & 0.001 & -0.015 \\
& -0.020 & -0.006 & -0.003 & -0.002 & -0.014 \\
& 0.022 & 0.007 & 0.003 & 0.002 & -0.002 \\
& -0.045 & -0.016 & -0.008 & -0.005 & -0.002
\end{tabular}

(b) Cotton

\begin{tabular}{c|ccccc} 
Time & \multicolumn{5}{|c}{ Risk Aversion } \\
Horizon & 1 & 3 & 6 & 10 & 1000 \\
\hline Original & 0.002 & 0.000 & -0.001 & -0.001 & -0.164 \\
& -0.002 & -0.001 & -0.001 & -0.002 & -0.164 \\
1.5 & 0.003 & 0.001 & 0.000 & 0.000 & -0.078 \\
& -0.004 & -0.001 & -0.001 & -0.001 & -0.078 \\
3 & 0.004 & 0.002 & 0.001 & 0.001 & -0.006 \\
& -0.004 & -0.001 & -0.001 & -0.000 & -0.006 \\
6 & 0.004 & 0.002 & 0.001 & 0.001 & -0.001 \\
& -0.005 & -0.002 & -0.001 & -0.000 & -0.001 \\
12 & 0.012 & 0.004 & 0.002 & 0.001 & 0.000 \\
& -0.012 & -0.004 & -0.002 & -0.001 & 0.000 \\
24 & 0.024 & 0.008 & 0.004 & 0.003 & 0.000 \\
& -0.024 & -0.008 & -0.004 & -0.002 & 0.000
\end{tabular}

(d) Crude Oil

Table 6: Out-of-sample hedging performance, measured using out-of-sample utility, for varying time horizons (months) and risk aversion.

Notes: Out-of-Sample utility performance over the period July 1998 to December 2010 is measured using the in-sample hedge ratios detailed in table 3. At each time horizon, the first line is the performance assuming an expected return of $+2 \%$ in calculating the hedge ratio, while the second line refers to an expected return of $-2 \%$. The data is transformed into different time horizons using the wavelet filter (LA8) up to a 24 month horizon. 